\title{
Monoamine oxidase-B (MAO-B) inhibitors: implications for disease-modification in Parkinson's disease
}

Kay Cheong Teo ${ }^{1}$ and Shu-Leong $\mathrm{Ho}^{1,2^{*}}$

\begin{abstract}
There is a substantial amount of evidence from experimental parkinsonian models to show the neuroprotective effects of monoamine oxidase-B (MAOB) inhibitors. They have been studied for their potential disease-modifying effects in Parkinson's disease (PD) for over 20 years in various clinical trials. This review provides a summary of the clinical trials and discusses the implications of their results in the context of disease-modification in PD. Earlier clinical trials on selegiline were confounded by symptomatic effects of this drug. Later clinical trials on rasagiline using delayed-start design provide newer insights in disease-modification in PD but success in achieving the aims of this strategy remain elusive due to obstacles, some of which may be insurmountable.
\end{abstract}

Keywords: Parkinson's disease, Monoamine oxidase-B inhibitors, Disease-modification, Neuroprotection, Selegiline, Rasagiline

\section{Introduction}

For the purposes of this review, the term "disease-modification" refers to a broad definition of therapies which can slow or favorably modify the progressive degenerative processes in dopaminergic and non-dopaminergic neurons associated with Parkinson's disease (PD). In this context, the term "neuroprotection" has been used almost synonymously with "disease-modification". Monoamine oxidase-B (MAOB) is an enzyme that is involved in dopamine metabolism (Figure 1). MAOB inhibitors, namely selegiline and rasagiline, have been studied extensively for disease-modification in PD. This review will cover the rationale of this therapeutic strategy, clinical trials on MAOB inhibitors, the debates and caveats of the conclusions of these studies, and discuss the issues in deciding whether newer monoamine oxidase- $B$ inhibitors such as rasagiline should be prescribed in the context of diseasemodification in PD.

The incidence of PD increases with age, and is associated with significant disability. Its prevalence in patients

\footnotetext{
* Correspondence: slho@hku.hk

'Division of Neurology, Department of Medicine, University of Hong Kong, Hong Kong, China

${ }^{2}$ Research Centre of Heart, Brain, Hormone and Healthy Aging (HBHA), University of Hong Kong, Hong Kong, China
}

over 50 years of age is projected to double in the ten most populous nations over the next two decades [1]. Classical motor features of PD such as bradykinesia, rigidity and tremor develop when about $50 \%$ of dopaminergic nigrostriatal neurons and about $80 \%$ striatal dopamine production are lost [2]. However, non-dopaminergic neurons pathways also undergo degeneration in $\mathrm{PD}$, including cholinergic, noradrenergic, serotonergic and GABAergic pathways, resulting in a variety of non-motor features such as dementia, psychosis, depression and apathy. Unlike motor features which respond well to dopaminergic therapies such as levodopa and dopamine agonists, non-motor features which have either no or little response to dopaminergic therapies, can be more disabling to the patient and have a far greater impact on the quality of life. As yet, there is no conclusive proof that current therapy can delay, halt or reverse the neuronal degeneration in PD. Existing drug treatment is associated with a gradual loss of efficacy and long term side effects. Although stereotactic deep brain stimulation can relieve some motor symptoms, other motor and non-dopaminergic features are not relieved. As symptom-onset in most PD patients occur in their early 60s, even if partial disease-modification can be achieved, the progression to severe disability may be 


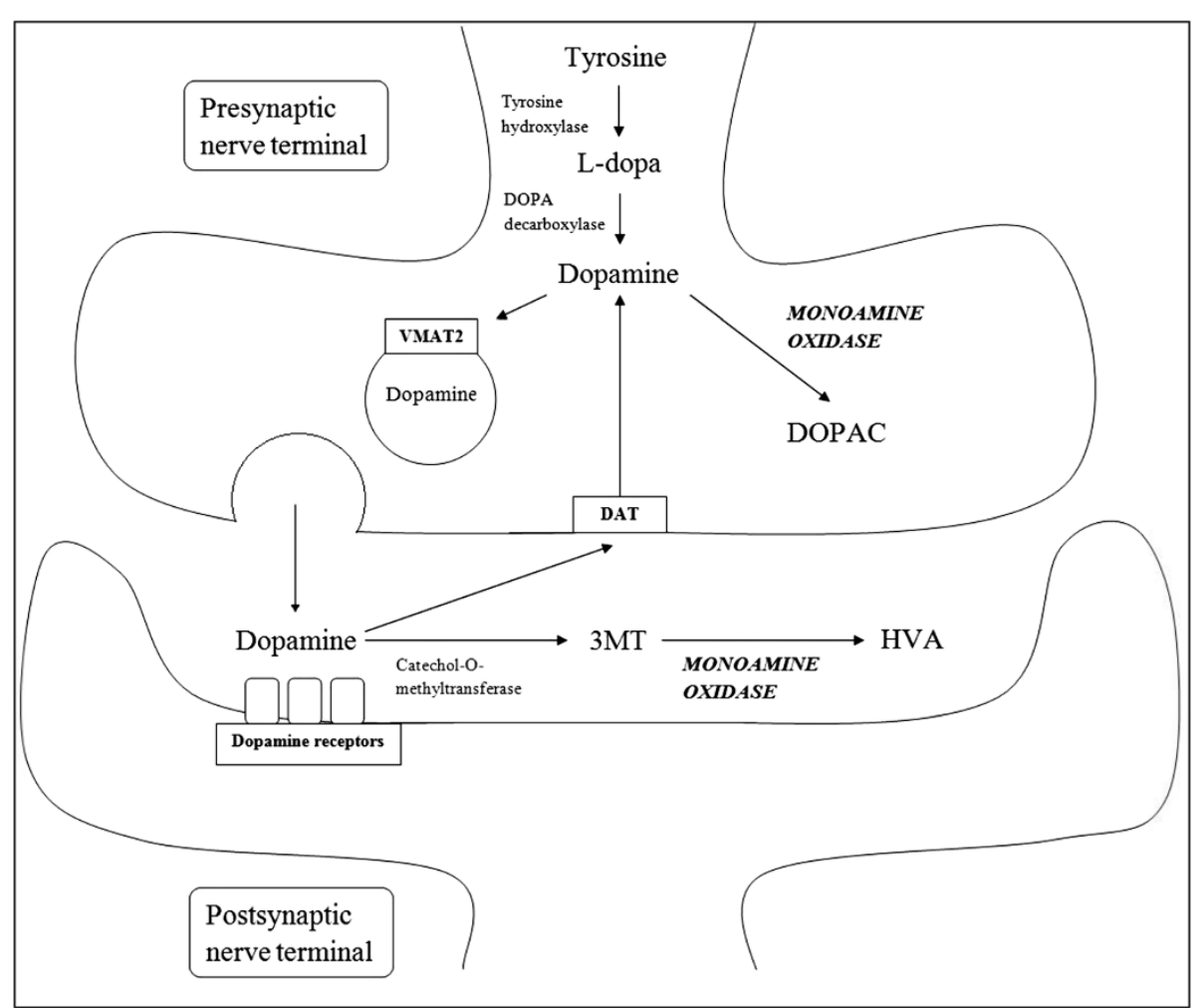

Figure 1 Dopamine metabolism and the action of monoamine oxidase. Abbreviations: VMAT2, vesicular monoamine transporter 2; DAT, dopamine active transporter; DOPAC, 3,4-dihydroxyphenylacetic acid; 3MT, 3-methoxytyramine; HVA, homovanillic acid.

delayed past the patients' natural life expectancy, so as to provide them with a satisfactory quality of life.

\section{Selegiline}

Selegiline (N-Propargyl-methamphetamine) is a selective, irreversible MAOB inhibitor at therapeutic dose of $10 \mathrm{mg} /$ day, but loses its selectivity at greater dosage [3]. Hence, selegiline at therapeutic dose does not cause the "cheese" reaction. The potential of selegiline to modify disease progression in PD was first proposed when it was shown to prevent 1-methyl-4-phenyl-1,2,3,6-tetrahydropyridine (MPTP)-induced parkinsonism in monkeys [4]. Invitro and invivo experiments have reported neuroprotective properties of selegiline [4-11]. Selegiline is a derivative of methamphetamine and is metabolized to L-amphetaminelike metabolites which can cause sympathomimetic side effects such as insomnia [12].

\section{Selegiline as monotherapy or an adjunct to levodopa}

Selegiline monotherapy was shown to provide modest symptomatic benefit and delay the need of levodopa therapy in early PD [13-15]. As an adjunct to levodopa therapy, selegiline can reduce motor fluctuations [15].

Deprenyl and Tocopherol Antioxidative Therapy of Parkinsonism (DATATOP) was the largest prospective controlled trial ever done for Selegiline [13]. The DATATOP study was initially designed to evaluate the neuroprotective properties of selegiline and tocopherol. Eight hundred untreated de novo PD patients were randomly assigned according to a $2 \times 2$ factorial design to one of the four treatment arms: selegiline placebo and alpha-tocopherol placebo; selegiline $10 \mathrm{mg} /$ day and alpha-tocopherol $2000 \mathrm{IU} /$ day; selegiline $10 \mathrm{mg} /$ day; and alpha-tocopherol $2000 \mathrm{IU} /$ day. Unified Parkinson's Disease Rating Scale (UPDRS) were evaluated at 1 month and 3 months after randomization, then approximately 3 monthly for a planned maximum of 2 years. The primary end point was reached when subjects developed a level of functional disability which required levodopa therapy. There was significant improvement of UPDRS score in the subjects who received selegiline during the 3 months "wash in" period indicating an early symptomatic benefit of selegiline. Selegiline delayed the need of levodopa by approximately 9 months. The Kaplan-Meier analysis showed that taking selegiline significantly reduced the probability of having to start levodopa therapy during the study period (hazard ratio 0.50; $95 \%$ confidence interval 0.41 to $0.62, \mathrm{p}<0.001)$. However, after a "wash out" period in subjects who did not reach the end point, there was a significant deterioration of the UPDRS score, indicating a symptomatic effect of selegiline. This symptomatic effect was not factored in during the initial study design. The results of DATATOP are 
generally considered as being significantly confounded by the symptomatic effects of selegiline.

Further evidence supporting the role of selegiline in the treatment of PD came from another multicentered, randomized, placebo-controlled, double-blinded study, involving 157 patients, who were randomly assigned to receive either selegiline $10 \mathrm{mg} /$ day or placebo [14]. The primary end point was reached when initiation of levodopa therapy became necessary. At 3 months follow up, the selegiline group had significant improvement of UPDRS total score $(-1.7 \pm 5.4$ vs. $1.0 \pm 5.3, \mathrm{p}<0.01)$, Visual Analogue Scale (VAS) tremor score $(-4.0 \pm 18.4$ vs. $4.0 \pm 16.9, \mathrm{p}<0.05)$ and VAS motor dysfunction score $(-3.0 \pm 21.3$ vs. $6.8 \pm 19.6$, $\mathrm{p}<0.05)$, when compared to the placebo group. The need for levodopa was delayed by 4.1 months with selegiline ( $\mathrm{p}=0.028)$. In their follow up study up to 7 years involving 141 patients, either selegiline or placebo was restarted in addition to levodopa therapy after an initial 8 weeks "wash out" period [16]. The selegiline group had slower disease deterioration as measured by the UPDRS total score $(\mathrm{p}=0.003)$, motor $(\mathrm{p}=0.002)$ and ADL $(\mathrm{p}=0.0002)$ subscores. Considering both the initial monotherapy and subsequent combination therapy up to 7 years, selegiline did not delay the start on wearing off fluctuations (hazard ratio 0.55 ; $95 \%$ confidence interval: 0.28 to $1.07, \mathrm{p}=0.076$ ).

A recent systemic review supported the early symptomatic and long term benefit of selegiline [15]. Selegiline was shown to be beneficial compared to control in motor impairment in 4 randomized control trials (RCTs) involving 986 patients. The weighted mean difference (WMD) for the change in motor UPDRS score was -4.49 (95\% confidence interval: -5.52 to -3.46$)$ and WMD in UPDRS ADL score was -2.19 ( $95 \%$ confidence interval: -2.78 to -1.60 ) at 1 year. Motor fluctuations were significantly reduced with selegiline (6 RCTs involving 1461 patients, odds ratio 0.73; $95 \%$ confidence interval: 0.58 to 0.91 ) at a mean weighted duration of follow up of 3.4 years. There was no significant difference in death or dyskinesia over the control subjects.

\section{Selegiline in clinical trials for disease-modification in PD}

There is no conclusive evidence from clinical trials to prove that selegiline has "disease-modification" effects, even though it was shown to have neuroprotective properties in invitro and invivo experimental models [4-11]. Long term clinical trials of selegiline have shown improved motor outcome and reduced levodopa requirement [16-19]. Whether these findings were attributed to the symptomatic benefits or the disease-modification property of selegiline remain debatable. Unlike rasagiline in which delayed-start design trials were carried out in an attempt to separate confounding symptomatic effects from disease-modifying effects, there are none for selegiline (discussed in more detail below).

\section{Lazabemide}

Lazabemide (N-(2-aminoethyl)-5-chloro-2-pyridinecarboxamide) was first tested in clinical trials for treatment of $\mathrm{PD}$ in the 1990s. Lazabemide is a more selective inhibitor of MAOB when compared to selegiline. Unlike selegiline, it is not metabolized to L-amphetamine-like metabolites and has a shorter "wash out" period [20]. The shorter "wash out" period of lazabemide was thought to provide a better assessment of the disease-modifying effects, as the change in PD status between baseline and post-treatment after a "wash out" period may not be confounded by any persistent symptomatic effect [21]. The disease-modifying effects of lazabemide was assessed in a randomized controlled trial which involved 321 de novo early PD patients [21]. Patients were randomly assigned to receive either placebo or lazabemide 25 to $200 \mathrm{mg} /$ day with a 2 to 4 week "wash out" period. Similar to selegiline, lazabemide was found to have a mild symptomatic effect. The probability of reaching disability sufficient to need initiation of levodopa therapy was significant reduced in lazabemide-treated patients (hazard ratio $0.49 ; 95 \%$ confidence interval 0.32 to 0.77; $\mathrm{p}=0.001$ ). After the "wash out" period, lazabemidetreated patients had a slower deterioration of PD impairment as evidenced by a lesser decline in total UPDRS score when compared to placebo ( $-6.1 \pm 8.4$ vs. $-8.1 \pm 8.5$; $\mathrm{p}=0.06$ ), indicating a possible disease-modifying effect. However, its manufacturer decided to halt further development of lazabemide in 1999 after reporting liver toxicity [22].

\section{Rasagiline}

Rasagiline (N-propargyl-1-(R)-aminoindan) is a second generation propargylamine-based selective, irreversible MAOB inhibitor. It was reported to have potent antiapoptotic effects independent of MAO inhibition in invitro and invivo experimental parkinsonian models [23-25]. It can cross the blood-brain-barrier readily [26]. Similar to selegiline, rasagiline lacks dietary tyramine sympathomimetic potentiation at its MAOB inhibitory dosage [23,27], and hence, lacks the undesirable pressor effects of the "cheese" reaction associated with MAOA inhibition. Unlike selegiline, rasagiline is not metabolized to L-amphetamine-like metabolites which may cause appetite suppression and insomnia [24].

\section{Rasagiline as an adjunct therapy to levodopa for motor fluctuations in PD}

Rasagiline was tested as an adjunct therapy to levodopa in alleviating parkinsonian motor fluctuations in two separate multicentered, double-blind, parallel-group clinical trials: a) PRESTO (Parkinson's Rasagiline: Efficacy and Safety in the Treatment of "Off") [28] and b) LARGO (Lasting effect in Adjunct therapy with Rasagiline Given Once daily) studies [29]. 
In the PRESTO study, $472 \mathrm{PD}$ patients with at least $2 \frac{1}{2}$ hours of daily "off" time were randomized to receive rasagiline (in either $1 \mathrm{mg} /$ day or $0.5 \mathrm{mg} /$ day) or placebo, as an adjunct therapy to levodopa over 26 weeks [28]. In its primary outcome measure, the mean adjusted total daily "off" time as measured by patients' home diaries had a modest but statistically significant reduction from baseline by 1.85 hours (29\%) in patients treated with $1 \mathrm{mg} /$ day dose, by 1.41 hours (23\%) with $0.5 \mathrm{mg} /$ day dose, and 0.91 hours (15\%) with placebo. Patients had 0.94 hours and 0.49 hours less "off" time on $1 \mathrm{mg} /$ day and $0.5 \mathrm{mg} /$ day doses respectively, compared to placebo. The changes on activities of daily living (ADL) and quality of life were less consistent. The Clinical Global Impression (CGI) and the UPDRS ADL scores during "off" time showed improvement as secondary end points with both doses of rasagiline, but not with PDQUALIF (PD Quality of Life) summary score.

In the LARGO study, $687 \mathrm{PD}$ patients with at least 1 hour of "off" time during awake hours as measured by 24-hour patients' home diaries, were randomized to receive either rasagiline (1 mg/day), entacapone (200 mg with each dose of levodopa) or placebo, as an adjunct therapy to levodopa for 18 weeks [29]. In its primary outcome measure, patients who had received rasagiline had a statistically significant reduction of mean daily "off" time by 1.18 hours; those on entacapone by 1.2 hours (both about $20 \%$ ), and placebo by 0.4 hours from baseline. The mean daily "on" time without troublesome dyskinesia had a modest but statistically significant increase of 0.85 hours in those patients who received rasagiline compared with 0.03 hours who received placebo; the improvement was similar in magnitude to entacapone. The CGI and UPDRS ADL scores during "off" time significantly improved in those patients taking rasagiline compared to placebo, comparable to entacapone.

In both the PRESTO and LARGO trials, rasagiline was well-tolerated. The adverse events which included nausea, anorexia and postural hypotension were mild. Rasagiline did not have any significant effects on the pulse or blood pressure.

\section{Rasagiline in clinical trials for disease-modification in PD} Apart from its effects on the motor symptoms of PD, rasagiline was tested for its neuroprotective effects in two prospective, double-blind, placebo-controlled, parallelgroup, randomized clinical studies on patients with early PD: a) TEMPO (TVP-1012 in Early Monotherapy for PD Outpatients) [30,31], and b) ADAGIO (Attenuation of Disease Progression with Azilect Given Once-daily) studies [32]. Patients with early stages of untreated PD were chosen because of the concern that the pathogenic processes and neuronal cell death would have been too far advanced in the late stages of PD for disease-modification to make any clinically meaningful impact. Another major concern raised after the conclusion of the DATATOP study and the subsequent studies on selegiline, was the confounding effects of symptomatic improvement on the assessment of disease-modifying benefits of the drug [13].

Both TEMPO and ADAGIO studies used a delayed-start design in an attempt to separate confounding symptomatic from disease-modifying effects [30-32]. The design essentially divided the trial period into two phases. The treatment groups would be randomized in the first phase to either placebo or rasagiline treatment arms. The first phase would have to be sufficiently long to allow any diseasemodifying effects of rasagiline more opportunity to manifest. This initial phase would be followed by a second phase where all the groups would receive rasagiline but the initial randomization was kept blinded. As all the treatment arms received rasagiline during the second phase of the study, any confounding symptomatic effects between the different arms at the end of the study would be negated. Hence, any differences in outcome would presumably be due to disease-modifying effects of rasagiline from the delayed start of rasagiline during the first phase of the study.

In the TEMPO study, 404 early PD patients who did not require dopaminergic therapy were randomized to three parallel arms: $1 \mathrm{mg} /$ day or $2 \mathrm{mg} /$ day for 12 months, or placebo for the first 6 months followed by rasagiline $2 \mathrm{mg} /$ day for another 6 months [30,31]. In its analysis based on the primary outcome measure over the first 6 month trial period, the total UPDRS score (adjusted for effect size) worsened significantly less compared to placebo from baseline; by 4.2 points less than placebo (95\% confidence interval: 5.66 to 2.73 points, $\mathrm{p}<0.001$ ) in patients who received rasagiline $1 \mathrm{mg} / \mathrm{day}$, and by 3.56 points less than placebo ( $95 \%$ confidence interval: 5.04 to 2.08 points, $\mathrm{p}<0.001$ ) in patients who received $2 \mathrm{mg} /$ day dose [30]. The improvement observed was similar between the two doses of rasagiline over this initial 6 month period. Although there are no direct comparisons as yet, the symptomatic effects of rasagiline monotherapy in early PD appear to be more modest than dopamine agonists. In a later analysis performed in the same study, patients who received rasagiline $1 \mathrm{mg} /$ day over 12 months had less worsening of mean adjusted total UPDRS score of 1.82 points (95\% confidence interval: 3.64 to 0.01 points, $\mathrm{p}=0.05$ ) compared with the delayed start rasagiline $2 \mathrm{mg} /$ day [31]. Patients who received rasagiline $2 \mathrm{mg} /$ day over 12 months had even less worsening of mean adjusted total UPDRS score of 2.29 points (95\% confidence interval: 4.11 to 0.48 points, $\mathrm{p}=0.01$ ) compared with the delayed start rasagiline $2 \mathrm{mg} /$ day. The study concluded that rasagiline at either $1 \mathrm{mg} /$ day or $2 \mathrm{mg} /$ day had less functional decline compared with the delayed start 
group. These studies raised the possibility that early-start rasagiline appeared to have enduring benefits over delayedstart, but it also raised concerns [33]. In an open-labeled extension the TEMPO study, 306 patients who continued on rasagiline were followed up to $6 \frac{1}{2}$ years (mean \pm SD: $3.6 \pm$ 2.1 years) [34]. The adjusted mean difference in change from baseline in total UPDRS scores was 2.5 points $(16 \%)$ in favor of early-start compared to delayed-start. This extension study also reported that although the interaction between treatment and time was significant, there was significantly less worsening in total UPDRS scores in the early-start compared to the delayed-start group at all seven half-yearly follow up time points.

The ADAGIO study which was larger and longer, but with a similar delayed-start design on rasagiline soon followed [35]. It involved 1,176 patients with early, untreated PD randomized in four parallel arms: rasagiline at either $1 \mathrm{mg} /$ day or $2 \mathrm{mg} /$ day for a total of 72 weeks (early-start group), and placebo for 36 weeks followed rasagiline at either $1 \mathrm{mg} /$ day or $2 \mathrm{mg} /$ day for another 36 weeks (delayed-start group). In order for the study to indicate significant disease-modification, the early-start group had to meet each of three hierarchical ends points of the primary analysis based on the total UPDRS score: a) superiority to placebo in the rate of change of the UPDRS score between weeks 12 and 36 during the first phase of the study, b) superiority to delayed-start treatment in the change of the score from baseline to week 72 , and c) non-inferiority to delayed-start treatment in the rate of change of the score between weeks 48 and 72 during the second phase of the study. The results showed that rasagiline $1 \mathrm{mg}$ /day dose achieved all three hierarchical primary endpoints based on disease progression: a) a slower mean rate of worsening in total UPDRS score between weeks 12 and 36 compared to placebo (0.09 points/ week in early-start treatment versus 0.14 points/week in placebo group), b) less worsening of mean total UPDRS score from baseline to week 72 in early-start ( 2.82 points) compared delayed-start (4.52 points) treatment, c) noninferiority of the early-start treatment in the mean rate of change of the total UPDRS score between weeks 48 and 72 in early-start (0.085 points/week) compared with delayed-start (0.085 points/week) treatment. However, the $2 \mathrm{mg} /$ day dose failed to achieve all its primary endpoints because the change of mean total UPDRS score from baseline to week 72 in the early-start (3.47 points) was not significantly different from the delay-start treatment (3.11 points). However, the early-start treatment group taking rasagiline $2 \mathrm{mg} /$ day dose had less mean rate of worsening (0.07 points/week) in total UPDRS score compared with placebo ( 0.14 points/week) between weeks 12 to 36 . Furthermore, at the $2 \mathrm{mg} /$ day dose, the mean rate of change in total UPDRS between weeks 48 to 72 in the early-start (0.094 points/week) was non-inferior to delayed-start treatment (0.065 points/week). Nevertheless, the result of the $2 \mathrm{mg} /$ day dose was considered to be negative because the design was such that all three hierarchical primary endpoints had to be met for each separate dose. The authors concluded that early-start treatment rasagiline at $1 \mathrm{mg} /$ day provided benefits consistent with possible disease-modifying effects even though it did not met its endpoints at $2 \mathrm{mg} /$ day.

The ADAGIO study results raised some debate and concerns, in particular, the divergent and paradoxical outcome between $1 \mathrm{mg} /$ day and $2 \mathrm{mg} /$ day doses [36,37]. It is unknown how PD progresses and whether it does so linearly. It was also unclear whether confounding symptomatic effects of rasagiline were significantly negated. The first hierarchical endpoint of superiority to placebo in the rate of change of the total UPDRS score between weeks 12 and 36 relied on the assumptions that, a) the rate of change of this score was linear during this period; and b) the symptomatic effects of rasagiline were fully established by week 12 [35]. This linearity from weeks 12 to 36 and whether the confounding symptomatic effects were fully established before the assessments on the first endpoint, have been disputed [36], and questions raised on whether the rasagiline $1 \mathrm{mg} /$ day dose had met the pre-specified criteria. The authors of the ADAGIO trial argued that there are many pharmacological examples where dose-related efficacy may not occur but it was thought that the doubling of the rasagiline dose was unlikely to have a $U$ or J curve effect $[38,39]$. The authors of the ADAGIO trial also noted that their posthoc analysis of the subgroup of patients with the highest total UPDRS score at baseline (i.e., the most severely affected quartile) had met all its primary endpoints with their early-start rasagiline $2 \mathrm{mg} /$ day dose [35]. They postulated that the results could be positive if the rasagiline $2 \mathrm{mg} /$ day dose was tested on more severely affected patients at baseline [39]. However, this could mean a higher drop-out rate especially in the placebo/active treatment group during the first phase of the study which could confound the results. Questions remain that if the trial was extended further in a blindedmanner during the second phase, whether the difference in outcome between the delayed-start and early-start treatment groups would diverge or converge.

\section{Symptomatic benefits of MAOB inhibitors in early PD when compared with other medications for PD}

MAOB inhibitors provide a modest symptomatic benefit in the treatment of early PD. The change in mean total UPDRS score after 3 months of selegiline $10 \mathrm{mg} /$ day was -1.6 points in the DATATOP study [13], while the change in mean score after 6 months of rasagiline $1 \mathrm{mg} /$ day was +0.1 points in the TEMPO study [30]. The deterioration in the UPDRS scores for the treatment groups in 




\footnotetext{
Compensatory changes for dopamine loss in presymptomatic Parkinson disease

1. Increase in corticostriatal excitatory connections [52].

2. Selectively reduce the excitatory connections to the indirect pathway [53].

3. Increase in the synthesis and release of dopamine [54].

4. Reduce in synaptic dopamine active transporter [55].

5. Increase in postsynaptic D2 receptor levels [56,57].

6. Reduce the ability to release GABA in the striatopallidal indirect pathway [53].

7. Increase in the synaptic strength of GPe-STN connections [58].

8. Hyperactivity of subthalamic nucleus [59].
}

Figure 2 Compensatory changes for dopamine loss in pre-symptomatic Parkinson's disease. Abbreviations: GPe, globus pallidus pars externa; GPi, globus pallidus pars interna; STN, subthalamic nucleus; SNc, substantia nigra pars compacta; GABA, gamma-aminobutyric acid. Solid arrows: excitatory glutamatergic connections; Dot arrows: inhibitory GABAergic connections; Dash dot arrow: dopaminergic connections.

both studies was significantly slower when compared to placebo, indicating a symptomatic benefit of MAOB inhibitors. Although there are no direct comparisons as yet, the symptomatic benefits of MAOB inhibitors were weaker when compared to dopamine agonist or levodopa. The change of mean motor UPDRS score was -4.5 points for ropinirole, -3.4 points for pramipexole and -7.3 points for levodopa after 6 months of treatment [40,41].
Obstacles to clinical trials in disease-modification for PD

There are inherent problems with PD which may form insurmountable barriers to disease-modifying strategies in clinical trials. There are no consistently reliable biological or neuroimaging markers in PD. As yet, the diagnosis of PD is still made clinically. There are no similar markers to correlate with disease progression. Its definition, progression and primary outcome measures are

Table 1 Pharmacology properties and therapeutic benefits of MAOB inhibitors in clinical use for Parkinson's disease

\begin{tabular}{|c|c|c|}
\hline & Selegiline & Rasagiline \\
\hline Recommended dosage & 10 mg daily & 1 mg daily \\
\hline Bioavailability & Unknown [61] & $36 \%[62]$ \\
\hline Half-life & 10 hours [61] & $0.6-2$ hours $[62]$ \\
\hline Metabolite & L-amphetamine like metabolites [61] & Aminoindan [62] \\
\hline Symptomatic monotherapy & Efficacious [15] & Efficacious $[30,35]$ \\
\hline \multirow[t]{3}{*}{ Adjunct to levodopa and treatment to motor complications } & Likely efficacious [15] & Efficacious $[28,29]$ \\
\hline & - Levodopa sparing effect & - Levodopa sparing effect \\
\hline & - Reduction in motor fluctuation & - Reduction in motor fluctuation \\
\hline Disease-modification & Insufficient evidence & Insufficient evidence \\
\hline
\end{tabular}


still determined by its clinical features in many trials. Furthermore, its clinical features are heterogeneous, from tremor-predominant to akinetic-rigid forms, younger onset patients with more dystonic features, and older onset patients with more dementia [42,43]. Its progression also varies between different patients, and may differ within the same patient at different points in time [44-46]. The UPDRS is inadequate to address the many complexities associated with PD symptomatology. This score is focused primarily on dopaminergic responsive symptoms, and is poorly adapted to help define and monitor non-motor features which are more disabling,

Table 2 Major trials for selegiline and rasagiline

\begin{tabular}{|c|c|c|c|}
\hline \multirow{2}{*}{$\begin{array}{l}\text { Selegiline } \\
\text { DATATOP } 1993 \text { [13] }\end{array}$} & & \multicolumn{2}{|l|}{ Rasagiline } \\
\hline & & TEMPO 2004 [31] & \\
\hline Study design & RCT selegiline vs. placebo & Study design & $\begin{array}{l}\text { Randomized, delayed start trial rasagiline for } \\
1 \text { year vs. } 6 \text { months placebo then } \\
6 \text { months rasagiline }\end{array}$ \\
\hline Participants & 800 patients & Participants & 404 patients \\
\hline Follow up period & 2 years & Follow up period & 1 year \\
\hline End point & Functional disability requiring levodopa & End point & Change in UPDRS score \\
\hline Major finding & $\begin{array}{l}\text { Need for levodopa delayed by } 9 \text { months in } \\
\text { the selegiline group }\end{array}$ & Major finding & $\begin{array}{l}\text { Slower disease deterioration in the early start } \\
\text { rasagiline group }\end{array}$ \\
\hline \multirow[t]{2}{*}{ Conclusion } & \multirow[t]{2}{*}{ Symptomatic benefit } & \multirow[t]{2}{*}{ Conclusion } & Symptomatic benefit \\
\hline & & & Possible disease-modifying effect \\
\hline \multicolumn{2}{|l|}{ SELEDO 1999 [19] } & \multicolumn{2}{|l|}{ PRESTO 2005 [28] } \\
\hline Study design & $\begin{array}{l}\text { RCT selegiline vs. placebo, as adjunct to } \\
\text { levodopa }\end{array}$ & Study design & $\begin{array}{l}\mathrm{RCT} \text { rasagiline vs. placebo, as adjunct } \\
\text { to levodopa }\end{array}$ \\
\hline Participants & 116 patients & Participants & 472 patients with daily off time \\
\hline Follow up period & 5 years & Follow up period & 26 weeks \\
\hline End point & Increase in $>50 \%$ of initial levodopa dose & End point & Total daily off time \\
\hline Major finding & $\begin{array}{l}\text { Primary end point delayed in the } \\
\text { selegiline group }\end{array}$ & Major finding & Less off time in the rasagiline group \\
\hline Conclusion & Symptomatic benefit as adjunct to levodopa & Conclusion & Symptomatic benefit as adjunct to levodopa \\
\hline \multicolumn{2}{|l|}{ Larsen 1999 [17] } & \multicolumn{2}{|l|}{ LARGO 2005 [29] } \\
\hline Study design & $\begin{array}{l}\text { RCT selegiline vs. placebo, as adjunct to } \\
\text { madopar. } 1 \text { month wash out of selegiline at } \\
\text { the end of study period }\end{array}$ & Study design & $\begin{array}{l}\mathrm{RCT} \text { rasagiline vs. entacapone vs. placebo, as } \\
\text { adjunct to levodopa }\end{array}$ \\
\hline Participants & 163 patients & Participants & 687 patients with daily off time \\
\hline Follow up period & 5 years & Follow up period & 18 weeks \\
\hline End point & $\begin{array}{l}\text { Levodopa requirement and deterioration of } \\
\text { UPDRS score }\end{array}$ & End point & Total daily off time \\
\hline Major finding & $\begin{array}{l}\text { Lower levodopa requirement and UPDRS } \\
\text { score in the selegiline group }\end{array}$ & Major finding & Less off time in the rasagiline group \\
\hline \multirow[t]{2}{*}{ Conclusion } & Symptomatic benefit as adjunct to madopar & \multirow[t]{2}{*}{ Conclusion } & \multirow[t]{2}{*}{ Symptomatic benefit as adjunct to levodopa } \\
\hline & Possible disease-modifying effect & & \\
\hline \multicolumn{2}{|l|}{ Palhagen 2006 [16] } & \multicolumn{2}{|l|}{ ADAGIO 2009 [35] } \\
\hline Study design & $\begin{array}{l}\text { RCT selegiline vs. placebo, as adjunct } \\
\text { to levodopa }\end{array}$ & Study design & $\begin{array}{l}\text { Randomized, delayed start trial rasagiline for } \\
72 \text { weeks vs. } 36 \text { weeks placebo then } \\
36 \text { weeks rasagiline }\end{array}$ \\
\hline Participants & 140 patients & Participants & 1176 patients \\
\hline Follow up period & 7 years & Follow up period & 72 weeks \\
\hline End point & Deterioration of UPDRS score & End point & $\begin{array}{l}\text { Three hierarchical end points to indicate } \\
\text { significant disease-modification }\end{array}$ \\
\hline Major finding & $\begin{array}{l}\text { Slower disease deterioration in the } \\
\text { selegiline group }\end{array}$ & Major finding & $\begin{array}{l}\text { Rasagiline } 1 \mathrm{mg} / \text { day achieved all three } \\
\text { hierarchical end points, but not in } 2 \mathrm{mg} / \text { day dose }\end{array}$ \\
\hline Conclusion & Symptomatic benefit as adjunct to levodopa & Conclusion & Possible disease-modifying effect \\
\hline
\end{tabular}


and less responsive to treatment. Various efforts to improve the UPDRS and new diagnostic criteria for nonmotor features are being established in an effort to address these concerns [47-49]. This task is made more difficult considering that PD patients can have fluctuations in motor and non-motor clinical features even within the same day. Perhaps the most difficult issue is that the cause or causes of PD are unknown. PD may have heterogeneous etiologies and probably multiple pathogenic pathways. Clinical trials using single or even double agents designed to modify the course of a homogeneous disorder may well never achieve its aim. Conducting clinical trials is expensive, especially when combinations of therapies are tested for extended periods. There is still no clear consensus on what constitutes a disease-modifying therapy as there are many caveats to its definition.

\section{Potential benefits of early treatment in normalizing compensatory mechanisms in PD}

Disease-modifying therapy should intuitively have the most impact at the earliest stages of the disease when there are still functional neuronal networks to be preserved. Indirect evidence from the ELLDOPA (Earlier versus Later Levodopa Therapy in Parkinson Disease) [50], and TEMPO trials have shown that symptomatic therapies started early in the disease may help to reduce the severity in the latter stages of the disease compared with a later start [34]. Schapira and Obeso had proposed that compensatory changes in the basal ganglia circuitries and thalamo-cortical projections occur in the earlier stages of PD to maintain its physiological motor function in response to a gradual deficit in striatal dopamine associated with degeneration of the nigrostriatal pathway [51]. Compensatory changes can occur at the nigrostriatal pathway and the basal ganglia circuit [52-59] (Figure 2). They further proposed when the dopaminergic deficit in PD surpasses the threshold of basal ganglia compensatory mechanisms, motor symptoms develop associated with hyperactivity in globus pallidum externa-subthalamic nucleus-globus pallidum interna pathway and loss of modulation for normal basal ganglia output. A major feature of this basal ganglia imbalance is potentially deleterious glutamatergic hyperactivity arising from the subthalamic nucleus, pedunculopontine nucleus, intralaminar thalamic nucleus, and corticostriatal projection, exacerbating excitotoxicity and other pathological mechanisms. Earlier restoration of the dopaminergic deficit with early symptomatic treatment may restore this imbalance to a more normal state, and reduce deleterious pathological mechanisms which can exacerbate the progression of the disease. It is unclear whether early symptomatic treatment is associated with long term disease-modifying effects. Their hypothesis indicates that symptomatic and disease-modifying effects in clinical trials are not mutually exclusive [60].

\section{Conclusions}

Tables 1 and 2 summarize the pharmacology properties, therapeutic benefits and major trials for selegiline and rasagiline. There is little to choose between selegiline and rasagiline, although the latter does not have amphetaminelike side effects which may cause appetite suppression and insomnia. Unlike the laboratory evidence, there is currently no conclusive proof from existing clinical trials that MAOB inhibitors have disease-modification effects on the natural history of PD. Questions remain whether the results of the disease-modification trials reflect genuine disease-modifying effects, or confounded by the limitations of the clinical trials and the measures of efficacy. However, the potential benefits of MAOB inhibitors with little or well-tolerated side effects may be useful in some patients with $\mathrm{PD}$, especially in younger patients with milder symptoms as monotherapy or as an adjunct to levodopa.

\section{Competing interests}

Both authors have no conflict of interest. The corresponding author is a subsection editor of Translation Neurodegeneration.

\section{Authors' contributions}

KC and SL drafted the manuscript. Both authors read and approved the final manuscript.

\section{Acknowledgements}

The authors' work is supported by the Henry G Leong Endowed Professorship and the Donation Fund for Neurology Research, University of Hong Kong.

\section{Received: 19 March 2013 Accepted: 29 August 2013}

Published: 8 September 2013

\section{References}

1. Dorsey ER, Constantinescu R, Thompson JP, et al: Projected number of people with Parkinson disease in the most populous nations, 2005 through 2030. Neurology 2007, 68:384-386.

2. Braak H, Del Tredici $K$, Rüb $U$, et al: Staging of brain pathology related to sporadic Parkinson's disease. Neurobiol Aging 2003, 24:197-211.

3. Riederer P, Youdim MB: Monoamine oxidase activity and monoamine metabolism in brains of parkinsonian patients treated with I-deprenyl. J Neurochemistry 1986, 46:1359-1365.

4. Cohen G, Pasik P, Cohen B, et al: Pargyline and deprenyl prevent the neurotoxicity of 1-methyl-4-phenyl-1,2,3,6-tetrahydropyridine (MPTP) in monkeys. Eur J Pharmacology 1984, 106:209-210.

5. Finnegan KT, Skratt JJ, Irwin I, et al: Protection against DSP-4-induced neurotoxicity by deprenyl is not related to its inhibition of MAO B. Eur $J$ Pharmacol 1990, 184:119-126.

6. Knollema S, Aukema W, Hom H, et al: L-deprenyl reduces brain damage in rats exposed to transient hypoxia-ischemia. Stroke 1995, 26:1883-1887

7. de la Cruz CP, Revilla E, Steffen V, et al: Protection of the aged substantia nigra of the rat against oxidative damage by (-)-deprenyl. $\mathrm{Br} J$ Pharmacol 1996, 117:1756-1760.

8. Munirathinam S, Lakshmana MK, Raju TR: (-) deprenyl attenuates aluminium induced neurotoxicity in primary cortical cultures. Neurodegeneration 1996, 5:161-167.

9. Mytilineou C, Radcliffe P, Leonardi EK, et al: L-deprenyl protects mesencephalic dopamine neurons from glutamate receptor-mediated toxicity in vitro. J Neurochem 1997, 68:33-39. 
10. Maruyama W, Takahashi T, Naoi M: (-)-Deprenyl protects human dopaminergic neuroblastoma SH-SY5Y cells from apoptosis induced by peroxynitrite and nitric oxide. J Neurochem 1998, 70:2510-2515.

11. Tatton WG, Chalmers-Redman RM, Ju WJ, et al: Propargylamines induce antiapoptotic new protein synthesis in serum- and nerve growth factor (NGF)-withdrawn, NGF-differentiated PC-12 cells. J Pharmacol Exp Ther 2002, 301:753-764

12. Kamada T, Chow T, Hiroi T, et al: Metabolism of selegiline hydrochloride, a selective monoamine b-type inhibitor, in human liver microsomes. Drug Metab Pharmacokinet 2002, 17:199-206.

13. Parkinson Study Group: Effects of tocopherol and deprenyl on the progression of disability in early Parkinson's disease. N Engl J Med 1993, 328:176-183.

14. Palhagen $\mathrm{S}$, Heinonen $\mathrm{EH}$, Hagglund J, et al: Selegiline delays the onset of disability in de novo parkinsonian patients. Neurology 1998, 51:520-525.

15. Turnbull K, Caslake R, Macleod A, et al: Monoamine oxidase B inhibitor for early Parkinson's disease. Cochrane Database Syst Rev 2005(3). 10.1002/14651858. CD004898.pub2

16. Palhagen S, Heinonen EH, Hagglund J, et al: Selegiline slows the progression of the symptoms of Parkinson disease. Neurology 2006, 66:1200-6.

17. Larsen JP, Boas J, Erdal JE, et al: Does selegiline modify the progression of early Parkinson's disease? Results from a five year study. Eur J of Neurol 1999, 6:539-47.

18. Shoulson I, Oakes D, Fahn S, et al: Impact of sustained Deprenyl (Selegiline) in levodopa treated Parkinson's disease: a randomized placebo-controlled extension of the deprenyl and tocopherol antioxidative therapy of Parkinsonism trial. Ann Neurol 2002, 51:604-12.

19. Przunztek H, Conrad B, Dichgans J, et al: SELEDO: a 5 years long term trial on effect of selegiline in early parkinsonian patients treated with levodopa. Eur J of Neurol 1999, 6:141-50.

20. LeWitt PA, Taylor DC: Protection against Parkinson's disease progression: clinical experience. Neurotherapeutics 2008, 5:210-25.

21. Parkinson Study Group: Effect of Lazabemide on the progression of disability in early parkinson's disease. Ann Neurol 1996, 40:99-107.

22. : The development of lazabemide ('Tempium'; Roche) has been stopped. React Wkly 1999, 772:3.

23. Finberg JP, Youdim MB: Pharmacological properties of the anti-Parkinson drug rasagiline; modification of endogenous brain amines, reserpine reversal, serotonergic and dopaminergic behaviours. Neuropharmacology 2002, 43:1110-8.

24. Chen JJ, Swope DM: Clinical pharmacology of rasagiline: a novel, secondgeneration propargylamine for the treatment of Parkinson disease. J Clin Pharmacol 2005, 45:878-94.

25. Weinreb O, Amit T, Bar-Am O, Youdim MB: Rasagiline: a novel anti-Parkinsonian monoamine oxidase-B inhibitor with neuroprotective activity. Prog Neurobiol 2010, 92:330-44.

26. Youdim MB, Gross A, Finberg JP: Rasagiline (N-propargyl-1R (+)-aminoindan), a selective and potent inhibitor of mitochondrial monoamine oxidase B. Br J Pharmacol 2001, 132:500-6.

27. Finberg JP, Youdim MB: Modification of blood pressure and nictitating membrane response to sympathetic amines by selective monoamine oxidase inhibitors, types A and B, in the cat. Br J Pharmacol 1985, 85:541-6.

28. Parkinson Study Group: A randomized placebo-controlled trial of rasagiline in levodopa-treated patients with Parkinson disease and motor fluctuations: the PRESTO study. Arch Neurol 2005, 62:241-8.

29. Rascol O, Brooks DJ, Melamed E, et al: LARGO study group. Rasagiline as an adjunct to levodopa in patients with Parkinson's disease and motor fluctuations (LARGO, lasting effect in adjunct therapy with rasagiline given once daily, study): a randomised, double-blind, parallel-group trial. Lancet 2005, 365:947-54.

30. Parkinson Study Group: A controlled trial of rasagiline in early Parkinson disease: the TEMPO Study. Arch Neurol 2002, 59:1937-43.

31. Parkinson Study Group: A controlled, randomized, delayed-start study of rasagiline in early Parkinson disease. Arch Neurol 2004, 61:561-6.

32. Olanow CW, Hauser RA, Jankovic J, et al: A randomized, double-blind, placebo-controlled, delayed start study to assess rasagiline as a disease modifying therapy in Parkinson's disease (the ADAGIO study): rationale, design, and baseline characteristics. Mov Disord 2008, 23:2194-201.

33. Shults CW: Reexamination of the TEMPO Study. Arch Neurol 2005, 62:1320.
34. Hauser RA, Lew MF, Hurtig HI, et al: TEMPO Open-label Study Group. Long-term outcome of early versus delayed rasagiline treatment in early Parkinson's disease. Mov Disord 2009, 24:564-73.

35. Olanow CW, Rascol O, Hauser $R$, et al: ADAGIO Study Investigators. A double-blind, delayed-start trial of rasagiline in Parkinson's disease. $N$ Engl J Med 2009, 361:1268-78.

36. Schwarzschild MA: Rasagiline in Parkinson's disease. N Engl J Med 2010, 362:658.

37. Ahlskog JE, Uitti RJ: Rasagiline, Parkinson neuroprotection, and delayed-start trials: still no satisfaction? Neurology 2010, 74:1143-8.

38. Youdim MB: Rasagiline in Parkinson's disease. N Engl J Med 2010 362:657-8.

39. Olanow CW, Rascol O: Rasagiline in Parkinson's disease. N Engl J Med 2010, 362:658-9.

40. Adler $\mathrm{CH}$, Sethi KD, Hauser RA, et al: Ropinirole for the treatment of early Parkinson's disease. The ropinirole study group. Neurology 1997, 49:393-9.

41. Parkinson Study Group: Pramipexole vs levodopa as initial treatment for Parkinson disease: A randomized controlled trial. JAMA 2000, 284:1931-8.

42. Schrag A, Ben-Shlomo Y, Brown R, et al: Young-onset Parkinson's disease revisited-clinical features, natural history, and mortality. Mov Disord 1998, 13:885-94.

43. Selikhova M, Williams DR, Kempster PA, et al: A clinico-pathological study of subtypes in Parkinson's disease. Brain 2009, 132:2947-57.

44. Louis ED, Tang MX, Cote $\mathrm{L}$, et al: Progression of parkinsonian signs in Parkinson disease. Arch Neurol 1999, 56:334-7

45. Goetz CG, Stebbins GT, Blasucci LM: Differential progression of motor impairment in levodopa-treated Parkinson's disease. Mov Disord 2000, 15:479-84

46. Jankovic J, Kapadia AS: Functional decline in Parkinson disease. Arch Neurol 2001, 58:1611-5.

47. Goetz CG, Tilley BC, Shaftman SR, et al: Movement disorder society UPDRS revision task force. Movement Disorder Society-sponsored revision of the Unified Parkinson's Disease Rating Scale (MDS-UPDRS): scale presentation and clinimetric testing results. Mov Disord 2008, 23:2129-70.

48. Ravina B, Marder K, Fernandez $\mathrm{HH}$, et al: Diagnostic criteria for psychosis in Parkinson's disease: report of an NINDS, NIMH work group. Mov Disord 2007, 22:1061-8.

49. Emre $M$, Aarsland $D$, Brown $R$, et al: Clinical diagnostic criteria for dementia associated with Parkinson's disease. Mov Disord 2007 22:1689-707.

50. Fahn S, Oakes D, Shoulson I, et al: Levodopa and the progression of Parkinson's disease. N Engl J Med 2004, 351:2498-508.

51. Schapira AH, Obeso J: Timing of treatment initiation in Parkinson's disease: a need for reappraisal? Ann Neurol 2006, 59:559-62.

52. Calabresi P, Mercuri NB, Sancesario G, et al: Electrophysiology of dopamine-denervated striatal neurons. Implications for Parkinson's disease. Brain 1993, 116:433-52.

53. Brotchie J, Fitzer-Attas C: Mechanisms compensating for dopamine loss in early Parkinson disease. Neurology 2009, 72(Suppl 2):S32-8

54. Zigmond MJ, Acheson AL, Stachowiak MK, et al: Neurochemical compensation after nigrostriatal bundle injury in an animal model of preclinical parkinsonism. Arch Neurol 1984, 41:856-61.

55. Adams JR, van Netten $H$, Schulzer $M$, et al: PET in LRRK2 mutations: comparison to sporadic Parkinson's disease and evidence for presymptomatic compensation. Brain 2005, 128:2777-85.

56. Graham WC, Crossman AR, Woodruff GN: Autoradiographic studies in animal models of hemi-parkinsonism reveal dopamine D2 but not D1 receptor supersensitivity. I. 6-OHDA lesions of ascending mesencephalic dopaminergic pathways in the rat. Brain Res 1990, 514:92-102.

57. Joyce JN, Marshall JF, Bankiewicz KS, et al: Hemiparkinsonism in a monkey after unilateral internal carotid artery infusion of 1-methyl-4-phenyl1,2,3,6-tetrahydropyridine (MPTP) is associated with regional ipsilateral changes in striatal dopamine D-2 receptor density. Brain Res 1986 382:360-4.

58. Fan KY, Bauferton J, Surmeier DJ, et al: Proliferation of external globus pallidus-subthalamic nucleus synapses following degeneration of midbrain dopamine neurons. J Neurosci 2012, 32:13718-28.

59. Obeso JA, Rodriguez-Oroz MC, Lanciego JL: el al. How does Parkinson's disease begin? The role of compensatory mechanisms. Trends Neurosci 2004, 27:125-7. 
60. Obeso JA, Schapira AH: Compensatory mechanisms in Parkinson's disease. Mov Disord 2009, 24:153-4.

61. Selegiline hydrochloride (package insert). Morgantown, West Virginia: Mylan Pharmaceuticals; 2009

62. Azilect (package insert). Kfar-Saba, Israel: Teva Pharmaceutical; 2008.

doi:10.1186/2047-9158-2-19

Cite this article as: Teo and Ho: Monoamine oxidase-B (MAO-B)

inhibitors: implications for disease-modification in Parkinson's disease.

Translational Neurodegeneration 2013 2:19

\section{Submit your next manuscript to BioMed Central} and take full advantage of:

- Convenient online submission

- Thorough peer review

- No space constraints or color figure charges

- Immediate publication on acceptance

- Inclusion in PubMed, CAS, Scopus and Google Scholar

- Research which is freely available for redistribution 